\title{
REGION ADAPTIVE ADJUSTMENT STRATEGY BASED ON INFORMATION ENTROPY FOR REMOTE SENSING IMAGE SEGMENTATION
}

\author{
X. L. Li ${ }^{1, *}$ J. S. Chen ${ }^{1}$ \\ ${ }^{1}$ Center for Geospatial Information, Shenzhen Institutes of Advanced Technology, Chinese Academy of Sciences, Shenzhen, China - \\ (xl.li2, js.chen)@siat.ac.cn
}

Commission IV, WG IV/3

KEY WORDS: Information Entropy; Coefficient of Variation; Boundary-fitting; Selection factor; Fuzzy Clustering; Image Segmentation;

\begin{abstract}
:
For the difficulty of boundary-fitting in region-based algorithms, a region adaptive adjustment strategy based on information entropy is proposed for remote sensing image segmentation. Considering the characteristics of imperfect blocks that cover two homogeneous regions, a selection factor constructed by the spectral coefficient of variation and the information entropy of prior probability representing neighborhood constraint is designed to find the imperfect blocks. Then, the selected imperfect block is split into four equal parts, and new blocks enjoy the same membership as the original block. The model parameters are updated based on the current tessellation. If the fuzzy clustering objective function decrease, the split operation is certainly accepted, otherwise, it will be accepted with a certain probability to avoid local optimum. Finally, the experiments on simulated and multi-spectral remote sensing images show that the proposed strategy can accurately locate the imperfect blocks and effectively fit the boundary of homogeneous regions.
\end{abstract}

\section{INTRODUCTION}

Image segmentation plays a crucial role in remote sensing image interpretation (Mi and Chen, 2020; Troyagalvis et al., 2015), its main task is to partition the image into a group of homogeneous regions and ensure that (1) the features of pixels in the region is homogeneous and the site of pixels are highly connected in image space (Zhang et al., 2020a), (2) the segmentation boundaries between two homogeneous regions are consistent with the truth boundaries ( $\mathrm{Li}$ et al., 2015). However, due to the influence of the same ground object with different spectra, high-quality remote sensing image segmentation is always the research of general interest (Löw et al., 2015).

There are many image segmentation methods, such as clustering-based (Gong et al., 2013; Memon and Lee, 2018), statistical-based (Drăguţ et al., 2014; Schmitt et al., 2014), and so on. Clustering-based methods group the pixels according to the dissimilarity between pixels and the clustering center. It is an adaptive iterative optimization algorithm, which is widely used in image processing (Lei et al., 2019). To describe the statistical distribution characteristics, the statistical-based methods are also proposed to improve noise immunity (Permute et al., 2006). Combining the advantages of the two methods, Chatzis and Varvarigou (2008) proposed a statistical-based fuzzy clustering algorithm (HMRF-FCM). The fuzzy theory can deal with the problem that overlapping pixels are difficult to distinguish. It assumes that the pixels in the homogeneous region follow Gaussian distribution, and the dissimilarity is modeled by the negative logarithm of the probability density function. Then, the fuzzy clustering objective function is constructed by weighted dissimilarity measure with membership and adding the KL (Kullback-Leibler ) entropy regularization term. HMRF-FCM has made great achievements in pixel-based segmentation algorithms. However, the ability to overcome noise is still limited (Zhao et al., 2017).

Recently, the region-based algorithm has become the mainstream in remote sensing image segmentation (Kotaridis and Lazaridou, 2021; Zhang et al., 2020b), where the simplest way of regionalization is to divide the image domain into a group of regular blocks (Wang et al., 2016). The regular blocks are difficult to fit the boundary effectively. To improve it, Wang et al. (2015) proposed splitting and merging operations under 他 the Bayesian segmentation framework. However, split or merged blocks are randomly selected, which is not focalization and generates a lot of redundant operations. To find the imperfect blocks purposefully and improve segmentation results, a region adaptive adjustment strategy is proposed in this paper. First, a selection factor constructed from the spectral and spatial aspect is modeled to distinguish imperfect and perfect blocks, where the spectral characteristic is modeled by the spectral coefficient of variation, the spatial characteristic is modeled by the information entropy of prior probability representing neighborhood constraint. Based on the fuzzy clustering segmentation framework, the selected block is divided into four equal parts, and the newly generated blocks inherit the membership of the original one. Finally, the iterative optimization operation is completed under the regionalized HMRF-FCM (Zhao et al., 2017) framework to obtain the optimal segmentation results.

* Corresponding author 


\section{METHODOLOGY}

\subsection{Region adaptive adjustment strategy}

Taking regular tessellation as an example, the image $\boldsymbol{Z}=\left\{\boldsymbol{z}_{i}\left(x_{i}\right.\right.$, $\left.\left.y_{i}\right): i=1, \ldots, n\right\}$ is divided into a series of regular blocks $\boldsymbol{B}=\left\{\boldsymbol{B}_{j}\right.$ $j=1, \ldots, m\}$, where $i$ and $j$ are the index of pixels and blocks, $n$ and $m$ are the number of pixels and blocks, $\left(x_{i}, y_{i}\right)$ and $z_{i}=\left(z_{i a}: a\right.$ $=1, \ldots, b)$ are the position and the spectral vector of pixel $i, a$ and $b$ are the index and the number of bands, respectively.

The visualization model of imperfect blocks is shown in Figure 1 , the black frame represents regular blocks, the red frame represents imperfect block, the green frame represents perfect block, the bold blue line represents the boundary of two homogeneous regions (yellow and pink). It can be seen that the characteristics can be described from two aspects of the block itself and the neighboring blocks. Comparing to perfect block $\boldsymbol{B}_{2}$, there are two kinds of spectral information in the imperfect block $\boldsymbol{B}_{1}$. It shows that the spectra of the imperfect block will be more discrete. To describe the degree of dispersion, the spectral coefficient of variation of the block is defined as,

$$
C_{j}=\frac{1}{\boldsymbol{\mu}_{(j)} \Sigma_{(j)}^{-1} \boldsymbol{\mu}_{(j)}^{\mathrm{T}}}
$$

Where $\boldsymbol{\mu}_{(j)}$ and $\boldsymbol{\Sigma}_{(j)}$ are the mean and covariance of spectra in block $j$.

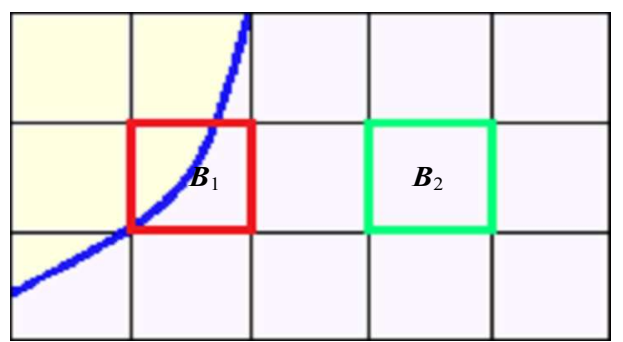

Figure 1. Comparison of the imperfect and perfect block.

For the spatial constraints aspect, the labels of neighboring blocks of the imperfect block are more diverse, and the segmentation uncertainty of this block is larger. It means that more information is needed to determine the label of the imperfect block. According to the theory of information entropy, the entropy of prior probability representing neighborhood label constraint is used to describe the diversity.

$$
E_{j}=-\sum_{l=1}^{k} p_{j l} \log p_{j l}
$$

Where $l$ and $k$ are the index and the number of clusters, respectively, $p_{j l}$ is the prior probability defined by neighborhood system $\boldsymbol{\Omega}=\left\{\boldsymbol{B}_{j},: j{ }^{\prime} \sim j\right\}, ' \sim$ ' represents the adjacency relation, $j$ ' is the index of neighboring blocks. To describe the spatial constraints, the prior probability is modeled based on Markov random field theory,

$$
p_{j l}=\frac{\exp \left[\delta \sum_{\boldsymbol{B}_{j^{\prime}} \in \boldsymbol{\Omega}_{j}} \eta\left(L_{j}=l, L_{j^{\prime}}\right)\right]}{\sum_{l^{\prime}=1}^{k} \exp \left[\delta \sum_{\boldsymbol{B}_{j^{\prime} \in \boldsymbol{\boldsymbol { \Omega } _ { j }}}} \eta\left(L_{j}=l^{\prime}, L_{j^{\prime}}\right)\right]}
$$

Where $L_{j}$ is the label of block $j, \delta$ is the neighborhood influence intensity, when $L_{j}=L_{j^{\prime}}, \eta\left(L_{j}, L_{j^{\prime}}\right)=1$, otherwise $\eta\left(L_{j}, L_{j^{\prime}}\right)=0$.

Combining the spectral coefficient of variation and information entropy of prior probability, the selection factor is defined as,

$$
S_{j}=C_{j} \times E_{j}
$$

The higher the value of $S_{j}$, the higher the degree of imperfection of the block. Then, the most imperfect block needed to be split is,

$$
h=\arg \max _{j=1, \ldots, m} S_{j}
$$

Block $B_{h}$ is chosen to split into four equal parts according to the operation, as shown in Figure 2, $c$ is the scale of the block. The newly generated blocks are renumbered counterclockwise as $m+1, m+2$, and $m+3$, and their membership is inherited from the original block, i.e. $u_{m+1 l}=u_{m+2 l}=u_{m+3 l}=u_{h l}$.

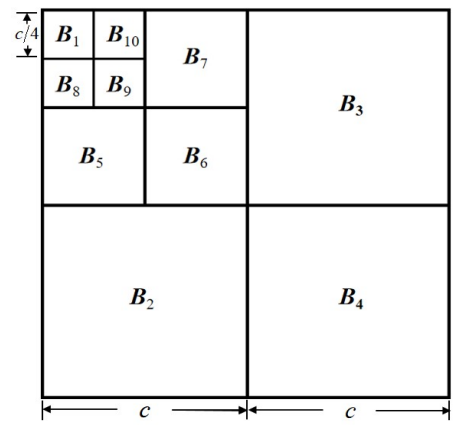

Figure 2. Splitting model.

\subsection{Fuzzy clustering segmentation}

Based on the block after regular tessellation, the region fuzzy clustering objection function is defined as (Zhao et al., 2017),

$$
J=\sum_{j=1}^{m} \sum_{l=1}^{k} u_{j l} d_{j l}+\lambda \sum_{j=1}^{m} \sum_{l=1}^{k} N_{j} u_{j l} \log \frac{u_{j l}}{p_{j l}}
$$

Where $u_{j l}$ is the membership of block $j$ belonging to cluster $l, \lambda$ is the coefficient of regularization term, $N_{j}$ is the number of pixels in block $j, d_{j l}$ is the dissimilarity measure defined by the negative logarithm of the probability density function of Gaussian distribution,

$$
\begin{aligned}
d_{j l}= & \sum_{\left(x_{i}, y_{i}\right) \in \boldsymbol{B}_{j}}-\log p\left(\boldsymbol{z}_{i} \mid \boldsymbol{\theta}_{l}\right)=\sum_{\left(x_{i}, y_{i}\right) \in \boldsymbol{B}_{j}}-\log \frac{1}{(2 \pi)^{b / 2}\left|\Sigma_{l}\right|^{1 / 2}} . \\
& \exp \left\{-\frac{1}{2}\left(\boldsymbol{z}_{i}-\boldsymbol{\mu}_{l}\right) \Sigma_{l}^{-1}\left(\boldsymbol{z}_{i}-\boldsymbol{\mu}_{l}\right)^{\mathrm{T}}\right\}
\end{aligned}
$$

Where $\boldsymbol{\theta}_{l}=\left\{\boldsymbol{\mu}_{l}, \boldsymbol{\Sigma}_{l}\right\}$ is the distribution parameter set, $\boldsymbol{\mu}_{l}$ and $\boldsymbol{\Sigma}_{l}$ are mean and covariance of the Gaussian distribution to which cluster $l$ follows.

Minimization of the objective function by derivative method, the analytical solution of $u_{j l}, \boldsymbol{\mu}_{l}$, and $\boldsymbol{\Sigma}_{l}$ can be obtained, 


$$
\begin{gathered}
u_{j l}=\frac{p_{j l} \exp \left(-\frac{1}{\lambda N_{j}} d_{j l}\right)}{\sum_{l^{\prime}=1}^{k} p_{j l^{\prime}} \exp \left(-\frac{1}{\lambda N_{j}} d_{j l^{\prime}}\right)} \\
\boldsymbol{\mu}_{l}=\frac{\sum_{j=1}^{m} u_{j l} \sum_{\left(x_{i}, y_{i}\right) \in \boldsymbol{B}_{j}} \boldsymbol{B}_{j}}{\sum_{j=1}^{m} N_{j} u_{j l}} \\
\sum_{l}=\frac{\sum_{j=1}^{m} u_{j l}\left(\boldsymbol{z}_{i}-\boldsymbol{\mu}_{l}\right)^{\mathrm{T}}\left(\boldsymbol{z}_{i}-\boldsymbol{\mu}_{l}\right)}{\sum_{j=1}^{m} N_{j} u_{j l}}
\end{gathered}
$$

Then, the optimal segmentation result can be obtained by maximum the optimal fuzzy membership $u_{j l}{ }^{*}$, i.e. $L_{j}=\arg \max _{l=1, \ldots, k} u_{j l}^{*}$.

\subsection{Summary of the proposed algorithm}

The process of the proposed algorithm can be summarized as follows,

S1: Initialization: Iteration index $t=0$; Scale of blocks $c$; Number of clusters $k$; Coefficient of regularization term $\lambda$; Neighborhood influence intensity $\delta$; Fuzzy membership $\boldsymbol{U}^{(0)}=\left[u_{j l}{ }^{(0)}\right]_{m \times k}$;

S2: Regular tessellation: $\boldsymbol{B}^{(t)}=\left\{\boldsymbol{B}_{j}^{(t)}: j=1, \ldots, m\right\}$;

S3: Regionalized fuzzy clustering image segmentation:

1. Calculating model parameters $\boldsymbol{\theta}_{l}^{(t)}=\left\{\boldsymbol{\mu}_{l}^{(t)}, \Sigma_{l}^{(t)}\right\}$ by Equations (9) and (10);

2. Calculating prior probability $p_{j l}^{(t)}$ by Equation (3);

3. Calculating dissimilarity measure $d_{j l}{ }^{(t)}$ by Equation (7);

4. Calculating fuzzy membership $u_{j l}{ }^{(t)}$ by Equation (8);

S4: Block splitting operation:

1. Calculating the spectral coefficient of variation $C_{j}^{(t)}$ by Equation (1) ;

2. Calculating the information entropy of prior probability $E_{j}^{(t)}$ by Equations (2) and (3);

3. Calculating the selection factor $S_{j}^{(t)}$ by Equation (4);

4. Finding the most imperfect block $h^{(t)}$ by Equation (5);

5. Splitting block $h^{(t)}$ according to Figure 2;

S5: Updating model parameters:

1. Calculating the region fuzzy clustering objection function $J^{*}$ by Equation (6);

2. If $J^{*}<J^{(t-1)}$, accept the splitting operation and updating model parameters; otherwise, accept it with a certain probability.

S6: Stop iteration until the objective function is minimum.

\section{EXPERIMENTS}

To highlight the effectiveness of the region adaptive adjustment strategy, the simulated and multi-spectral remote sensing images are tested by two comparison algorithms, HMRF-FCM (Chatzis and Varvarigou, 2008) and the regular tessellation fuzzy clustering algorithm, abbreviated as RT-HMRF-FCM (Zhao et al., 2017). The performance of the proposed algorithm is evaluated qualitatively and quantitatively.

Figure 3 is the simulated image and its segmentation results, where Figure 3(a1) is the simulated image, Figure 3(a2) and (a3) are the segmentation result and the outline overlay image of HMRF-FCM, Figure 3(b1)-(b3) are the regular tessellation result, segmentation result and outline overlay image of RTHMRF-FCM, Figure 3(c1)-(c3) are the adaptive regular tessellation result, segmentation result and outline overlay image of the proposed algorithm. It shows that there are many speckle mis-segmentation pixels because of the pixel-based mechanism of HMRF-FCM, as shown in Figures 3(a2) and (a3). The speckle mis-segmentation pixels will increase with spectral complexity. Although RT-HMRF-FCM can effectively overcome the speckle noise, the boundaries between homogeneous regions cannot be fitted smoothly, as shown in Figure 3(b1)-(b3). Comparing Figure 3(b1) and (c1), it can be seen that the imperfect blocks chosen to split are generally distributed on the boundaries of homogeneous regions, which advantageously verifies the effectiveness of the selection factor. Besides, the boundaries are fitted smoothly, which can verify the correctness of the combination of region adaptive adjustment strategy and fuzzy clustering segmentation. The proposed algorithm not only can well fit the boundary, but also avoid speckle noise.

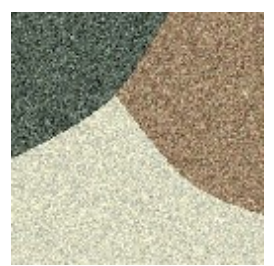

(a1)

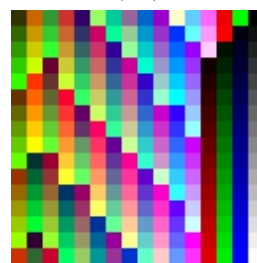

(b1)

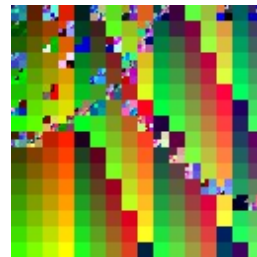

(c1)

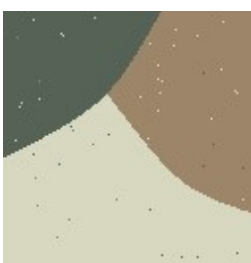

(a2)

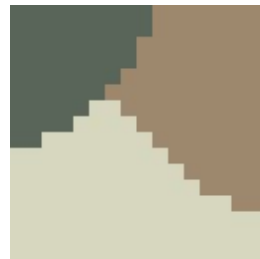

(b2)

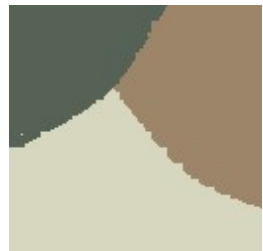

(c2)

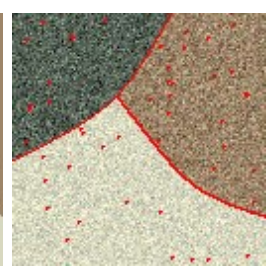

(a3)

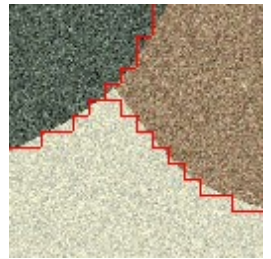

(b3)

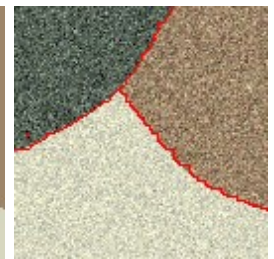

(c3)
Figure 3. Simulated image and its segmentation results. (a1) simulated image, (a2)-(a3) HMRF-FCM, (b1)-(b3) RT-

HMRF-FCM, (c1)-(c3) The proposed algorithm

To further demonstrate the applicability of the proposed algorithm, a lot of multi-spectral remote sensing images are also tested. The representative ones are shown in Figures 4 and 5. Figure 4(a1) is SPOT images, Figure 5(a1) is IKONOS image, Figure 4,5 (a2) and (a3) are the segmentation result and the outline overlay image of HMRF-FCM, Figure 4,5(b1)-(b3) are the regular tessellation results, segmentation result and outline 
overlay image of RT-HMRF-FCM, Figure 4,5(c1)-(c3) are the adaptive regular tessellation result, segmentation result and outline overlay image of the proposed algorithm.

In Figure 4, the segmentation results of the HMRF-FCM algorithm is seriously affected by the forest with big spectral variance, as shown in Figure 4(a2) and (a3). For RT-HMRF$\mathrm{FCM}$, the inner area of homogeneous regions is effectively segmented. However, the boundaries are extremely rough, such as the boundary between farm and forest, as shown in Figure 4(b2) and (b3). The proposed algorithm, benefited by the proposed adaptive selection and splitting strategy, can realize the smooth fitting of boundaries as well as the effective segmentation, as shown in Figure 4(c1) - (c3).

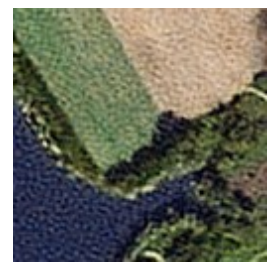

(a1)

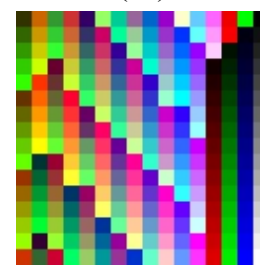

(b1)

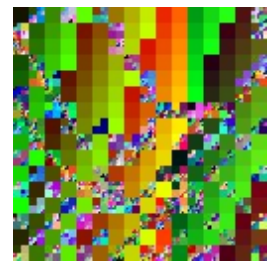

(c1)

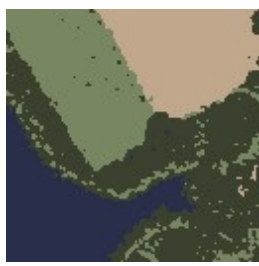

(a2)

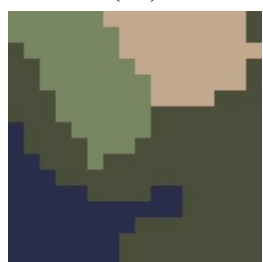

(b2)

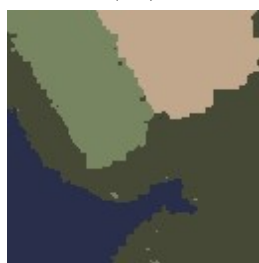

(c2)

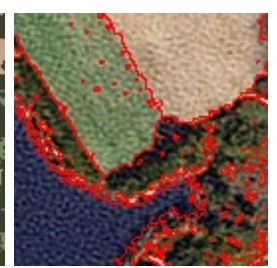

(a3)

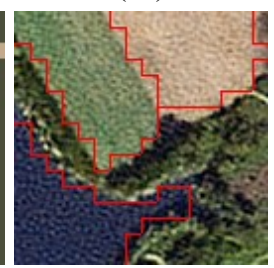

(b3)

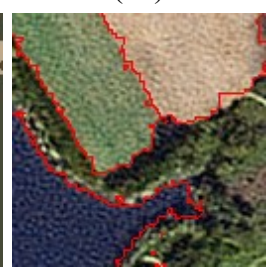

(c3)

Figure 4. SPOT image and its segmentation results. (a1) SPOT image, (a2)-(a3) HMRF-FCM, (b1)-(b3) RT-HMRFFCM, (c1)-(c3) The proposed algorithm

In Figure 5, there are high variance forests and thin roads. HMRF-FCM seriously confuses the forest with the grassland, as shown in Figures 5(a2) and (a3). RT-HMRF-FCM can not recognize the thin roads, and there are many mis-segmentation blocks in rough boundaries, as shown in Figure 5(b2) and (b3). From Figure 5(c1) - (c3), it can be seen that the proposed algorithm has a good ability in finding imperfect blocks and reserving perfect blocks, the segmentation result is better than others.

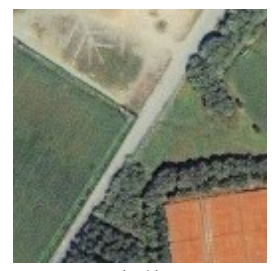

(a1)

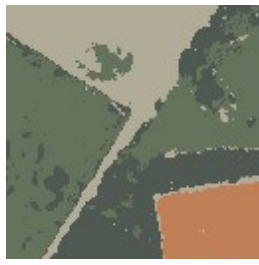

(a2)

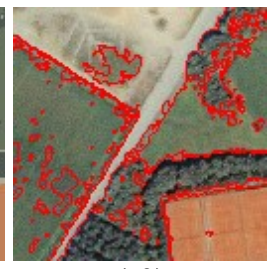

(a3)

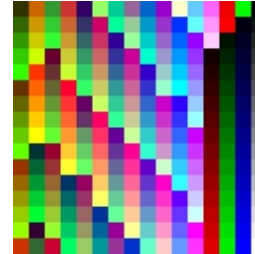

(b1)

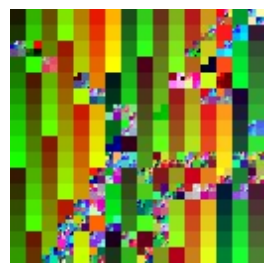

(c1)

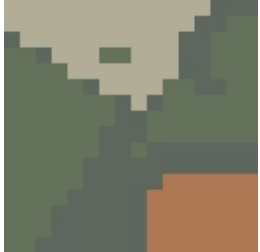

(b2)

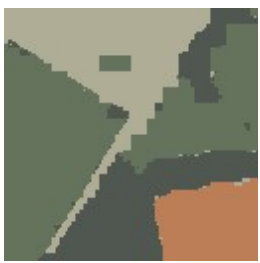

(c2)

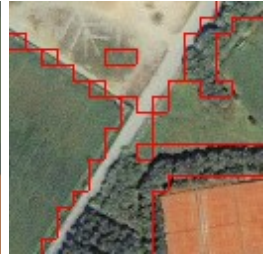

(b3)

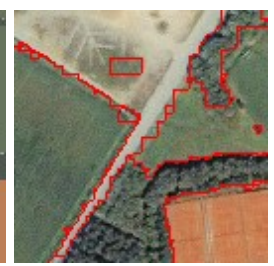

(c3)
Figure 5. IKONOS image and its segmentation results. (a1) IKONOS image, (a2)-(a3) HMRF-FCM, (b1)-(b3) RTHMRF-FCM, (c1)-(c3) The proposed algorithm

Figure 6 is buffer area analysis by constructing buffer for segmentation boundary and truth boundary, where columns 1-3 represent HMRF-FCM, RT-HMRF-FCM and the proposed algorithm, rows 1,3 , and 5 are the comparison of segmentation boundary and the buffer area of truth boundary, rows 2,4 , and 6 are the comparison of truth boundary and the buffer area of segmentation boundary. The green is right pixels, the red in Figure 6(a1)-(a3), (c1)-(c3) and (e1)-(e3) represents the redundant boundary in segmentation results, the red in Figure 6(b1)-(b3), (d1)-(d3) and (f1)-(f3) represents the deficient boundary in segmentation results. It can be seen that both redundant and deficient boundaries of the proposed algorithm segmentation results are the least compared with others.

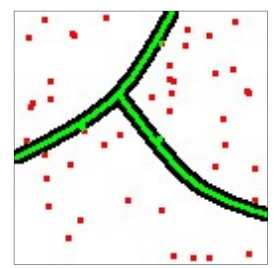

(a1)

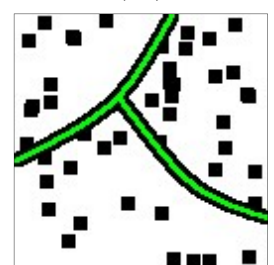

(b1)

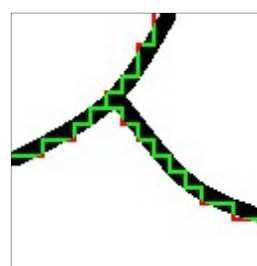

(a2)
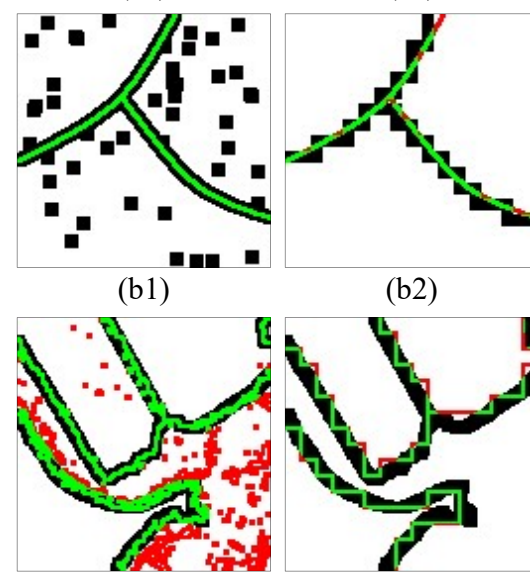

(c1)

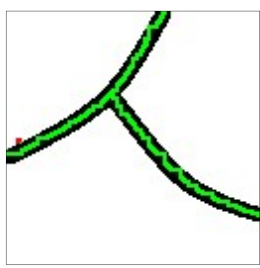

(a3)

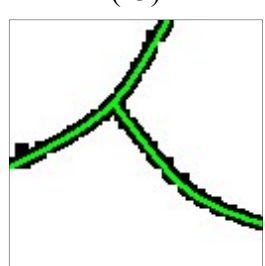

(b3) (b2)

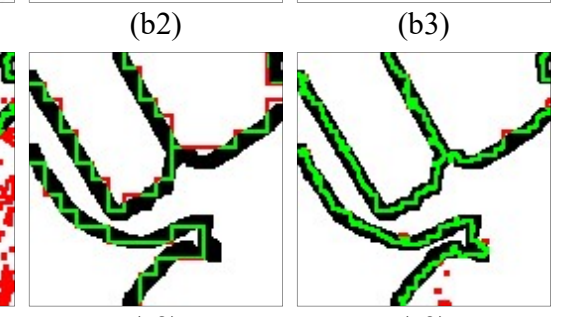

(c2)

(c3) 


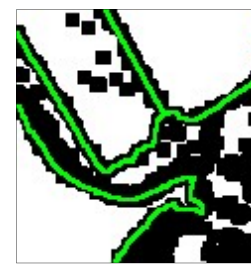

(d1)

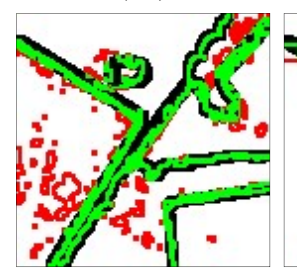

(e1)

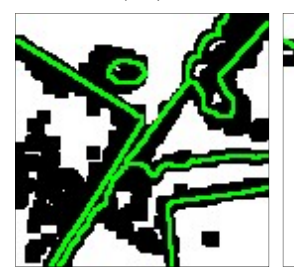

(f1)

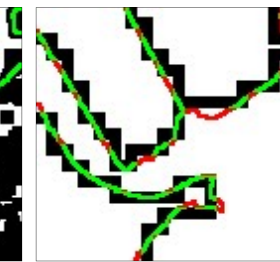

(d2)

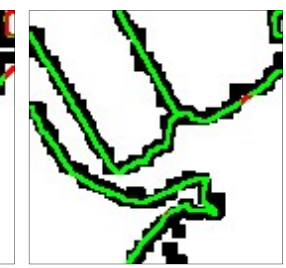

(d3)

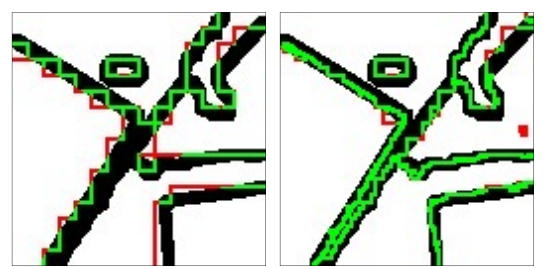

(e3)

(e2)

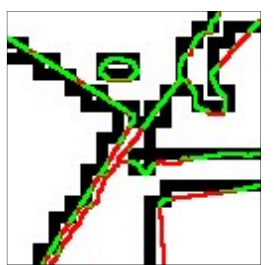

(f2)

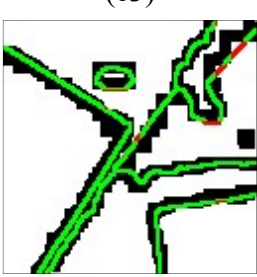

(f3)

Figure 6. Buffer area analysis of segmentation boundaries. (a1)-(f1) HMRF-FCM, (a2)-(f2) RT-HMRF-FCM, (a3)-(f3) The proposed algorithm.

Table 1 is the quantitative analysis, where User's accuracy (UA) is the ratio of the number of overlap pixels between segmentation boundary and truth boundary buffer area and the number of segmentation boundary pixels, Producer's accuracy (PA) is the ratio of the number of overlap pixels between truth boundary and segmentation boundary buffer area and the number of truth boundary pixels. It shows that the UA of HMRF-FCM tends to be low, such as the SPOT image is only $48.67 \%$. Although the UA of RT-HMRF-FCM has been improved, PA is decreased significantly, such as the PA of IKONOS is only $60.78 \%$. For the proposed algorithm, PA has almost no reduction with improving UA, both UA and PA are over $90 \%$.

\begin{tabular}{ccccc}
\hline \multirow{2}{*}{ Images } & \multirow{2}{*}{$\begin{array}{c}\text { Accuracy } \\
(\%)\end{array}$} & \multicolumn{3}{c}{ Algorithms } \\
\cline { 3 - 5 } & HMRF-FCM & $\begin{array}{c}\text { RT-HMRF- } \\
\text { FCM }\end{array}$ & $\begin{array}{c}\text { Proposed } \\
\text { algorithm }\end{array}$ \\
\hline \multirow{2}{*}{ Simulated } & UA & 61.44 & 85.12 & 98.63 \\
& PA & 100 & 81.80 & 100 \\
\multirow{2}{*}{ SPOT } & UA & 48.67 & 74.22 & 94.38 \\
& PA & 100 & 68.28 & 98.95 \\
IKONOS & UA & 60.49 & 65.92 & 90.79 \\
& PA & 99.93 & 60.78 & 93.27 \\
\hline
\end{tabular}

Table 1. Quantitative analysis

\section{CONCLUSION}

The pixel-based segmentation has weak noise immunity. However, the region-based algorithms are difficult to fit the boundary smoothly. Focus on the problem, a region adaptive adjustment strategy is proposed in this paper. By analyzing the characteristics of imperfect blocks that cover two or more homogeneous regions, the spectral coefficient of variation based on the block itself and the information entropy of the neighborhood system are modeled to find the imperfect blocks. The most imperfect block is chosen to split into four equal parts.

To avoid local optimum, the split operation will be accepted with a certain probability. A lot of experiments show that the proposed algorithm can find the imperfect blocks accurately, realize the effective segmentation and fit the boundaries smoothly. In the future, other strategies such as merging will be further studied to enhance the performance.

\section{ACKNOWLEDGEMENTS}

This research was funded by the Strategic Priority Research Program of the Chinese Academy of Sciences, grant number XDA19030301, and the National Natural Science Foundation of China, grant number (42001286).

\section{REFERENCES}

Chatzis, S. P., Varvarigou, T. A. 2008. A fuzzy clustering approach toward hidden Markov random field models for enhanced spatially constrained image segmentation. IEEE Transactions on Fuzzy Systems, 165, 1351-1361.

Drăguţ, L., Csillik, O., Eisank, C., Tiede, D. 2014. Automated parameterisation for multi-scale image segmentation on multiple layers. ISPRS Journal of photogrammetry and Remote Sensing, 88, 119-127.

Gong, M., Liang, Y., Shi, J., Ma, W., Ma, J. 2013. Fuzzy cmeans clustering with local information and kernel metric for image segmentation. IEEE transactions on image processing, 222, 573-584.

Kotaridis, I., Lazaridou, M. 2021. Remote sensing image segmentation advances: A meta-analysis. ISPRS Journal of Photogrammetry and Remote Sensing, 173, 309-322.

Lei, T., Jia, X., Zhang, Y., Liu, S., Meng, H., Nandi, A. K. 2019 Superpixel-based fast fuzzy C-means clustering for color image segmentation. IEEE Transactions on Fuzzy Systems, 279, 17531766.

Li, Z., Yang, Z., Xiong, H. 2015, November. Homogeneous region segmentation for SAR images based on two steps segmentation algorithm. In 2015 International Conference on Computers, Communications, and Systems ICCCS, pp. 196-200.

Löw, F., Knöfel, P., Conrad, C. 2015. Analysis of uncertainty in multi-temporal object-based classification. ISPRS Journal of Photogrammetry and Remote Sensing, 105, 91-106.

Memon, K. H., Lee, D. H. 2018. Generalised kernel weighted fuzzy C-means clustering algorithm with local information. Fuzzy Sets and Systems, 340, 91-108.

Mi, L., Chen, Z. 2020. Superpixel-enhanced deep neural forest for remote sensing image semantic segmentation. ISPRS Journal of Photogrammetry and Remote Sensing, 159, 140-152.

Permuter, H., Francos, J., Jermyn, I. 2006. A study of Gaussian mixture models of color and texture features for image classification and segmentation. Pattern Recognition, 394, 695706.

Schmitt, A., Wessel, B., Roth, A. 2014. An innovative curveletonly-based approach for automated change detection in multitemporal SAR imagery. Remote Sensing, 63, 2435-2462. 
Troya-Galvis, A., Gançarski, P., Passat, N., Berti-Equille, L. 2015. Unsupervised quantification of under-and oversegmentation for object-based remote sensing image analysis. IEEE Journal of Selected Topics in Applied Earth Observations and Remote Sensing, 85, 1936-1945.

Wang, Y., Li, Y., Zhao, Q. 2015. Segmentation of highresolution SAR image with unknown number of classes based on regular tessellation and RJMCMC algorithm. International Journal of Remote Sensing, 365, 1290-1306.

Wang, Y., Li, Y., Zhao, Q. 2016. Coupling regular tessellation with RJMCMC algorithm to segment SAR image with unknown number of classes. International Archives of the Photogrammetry, Remote Sensing and Spatial Information Sciences, XLI-B7, 393-397.

Zhang, X., Xiao, P., Feng, X. 2020a. Object-specific optimization of hierarchical multiscale segmentations for highspatial resolution remote sensing images. ISPRS Journal of Photogrammetry and Remote Sensing, 159, 308-321.

Zhang, Y., Liu, K., Dong, Y., Wu, K., Hu, X. 2020b. Semisupervised classification based on SLIC segmentation for hyperspectral image. IEEE Geoscience and Remote Sensing Letters, 17(8), 1440-1444.

Zhao, Q. H., Li, X. L., Li, Y., Zhao, X. M. 2017. A fuzzy clustering image segmentation algorithm based on hidden Markov random field models and Voronoi tessellation. Pattern Recognition Letters, 85, 49-55. 